\title{
FÓRMULAS ELECTORALES: UNA APLICACIÓN PRÁCTICA
}

Por: Teresa Blanco Hernández *

\section{Resumen}

En el presente artículo se analizan dos elementos del régimen electoral, como son las elecciones, la expresión del voto, y las distintas fórmulas repartidoras o de conversión de votos en escaños. En una segunda parte, de marcado carácter práctico, se aplican las diferentes fórmulas repartidoras proporcionales y sus variantes, a unos resultados electorales concretos y reales, mostrando la diferente distribución final de escaños entre los partidos políticos.

\section{ALABRAS CLAVE}

Elecciones, fórmula de reparto, fórmulas mayoritarias, fórmulas distributivas.

\section{INDUSTRIAL}

Los sistemas electorales, por su propia naturaleza, se consideran herramientas al servicio de la medición de las aspiraciones sociales. Sin embargo, en la actualidad, parece más acertado definirlos como instrumentos susceptibles de ser manejados por conveniencias partidistas. En palabras del profesor Vallés, "considerar el sistema electoral como instrumento científico de acción neutra y protegido de los vaivenes políticos sería desconocer que la estructura del proceso electoral está a su vez condicionada e influida por el contexto en que se inserta".(Vallés y Bosch, 1997:14).

Por otra parte, nos inquieta lo que algunos no han dudado en denominar "crisis de la democracia representativa", entendida como aquella que surge de la superación del Estado liberal para convertirse en un Estado social y democrático y cuyo objetivo no es otro sino el

\footnotetext{
* Doctora en Administración y Dirección de Empresas. Profesora de Economía Financiera.
} 
de velar por la protección de los derechos sociales y la provisión de servicios. Estas críticas respecto al funcionamiento de la misma $y$, en contra de lo que pudiera parecer, no resultan anecdóticas o puntuales, sino que, por el contrario, la han acompañado desde su inicio, debido, en gran medida, a que se trata de un sistema político abierto a la crítica.

Este debate se ha visto intensificado en un momento un tanto especial, puesto que la democracia liberal representativa se ha impuesto en países donde era desconocida hasta entonces o donde había tenido de ella experiencias muy fugaces o intermitentes. Así pues, podemos señalar a este respecto que, paradójicamente cuando menos viable parece una alternativa teórica a la democracia liberal, rebrota un cierto, llamemos, malestar democrático'.

La percepción de una amplia mayoría en el conjunto de agentes sociales es que se ha abierto una amplia brecha entre el ideal democrático para un horizonte temporal determinado y la práctica política. En otras palabras, hay un sentimiento generalizado de que la acción política democrática está desligada de las preocupaciones e intereses de la ciudadanía y, es por ello, que tanto científicos sociales como dirigentes políticos, se han esforzado y se esfuerzan en proponer reformas de tipo institucional, con el fin de modificar la configuración del sistema e introducir nuevos recursos tecnológicos en la esfera política.

Uno de los elementos clave y más repetido en dichas propuestas apunta a las formas de

1 Los indicadores que dan pie para afirmar sus existencia son de diverso orden: descrédito de los profesionales de la política, desinterés por la competición política, descenso de la militancia en partidos y organizaciones sociales, esquematización publicitaria del debate político,....(Valles y Bosch, 1997: 28).

\section{A}

This article analyzes two elements of the electoral process; what an election is like in terms of voter expression, and the diverse formulas which convert votes into seats. In addition, and on a more practical note, how the application of these proportion formulas and their variations, find the true electoral results while also disclosing the distribution of seats among the political parties.

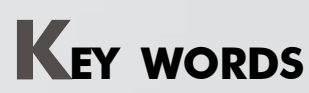

Elections, formulas of distribution, majority formulas, distributive formulas. 
participación ciudadana y en concreto a los sistemas electorales. En ellas, se hace especial mención a la importancia del referéndum para,, de esta manera, conseguir una intervención directa del ciudadano en la decisión política y complementar y /o compensar las insuficiencias de la intervención electoral.

Otras propuestas, con una perspectiva más tradicional, se refieren a las modificaciones de algunos elementos de los sistemas electorales, tal y como hoy los conocemos, y que son objeto de este artículo.

Pero antes de adentrarnos en el estudio de dichos elementos, es preciso detenernos en la definición de tres conceptos, en forma de tres preguntas, empezando por la más general para llegar a la más particular, teniendo en cuenta las palabras del profesor González:

¿Qué es el Derecho Electoral?

¿Qué es la Ley Electoral?

¿Qué es el Régimen Electoral?

\section{¿Qué es el Derecho Electoral?}

Es "un derecho sustantivo, propio y esencial del ordenamiento público estatal, que configura el carácter democrático del Estado y determina el grado de legitimidad que alcanzan los órganos constitucionales y las instituciones políticas de carácter representativo que lo integran". (González: 1996, 19).

\section{¿Qué se entiende por Ley Electoral?}

Es "la norma primaria de un Estado democrático en cuanto que constituye un marco garantizador de la libre competencia por el poder entre las fuerzas políticas en presencia, cuya articulación procedimental es la elección como método especial de selección de los dirigentes y por ende de creación de los órganos representativos" (González: 1996, 18)

\section{¿Qué es el Régimen Electoral?}

Es "un procedimiento mediante el cual se articula jurídicamente el proceso político de las elecciones democráticas, dando respuestas técnico-positivas y solucionando normativamente los problemas que aquellas comportan". (González: 1996, 19)

Y dentro de este último concepto, conviene señalar los tres elementos fundamentales del régimen o sistema electoral²:

- Circunscripción electoral.

- La forma de expresión del voto.

- La fórmula repartidora o la conversión de votos en escaños.

2 Arnaldo, 1999.34. 


\section{LA FORMA DE EXPRESIÓN DEL VOTO}

Ua forma de expresión del voto y, más en concreto las elecciones, ostenta la consideración de elemento clave del Estado democrático liberal, tal y como ha sido definido previamente. Al mismo tiempo, el principio democrático se proyecta de manera inmediata en las elecciones: su centralidad en el sistema democrático las convierte no sólo en una exigencia funcional imprescindible, sino en exponente de la verdadera condición de Estado democrático.

Con el fin de corroborar esta afirmación, basta detenerse en la Declaración Universal de los Derechos Humanos de 1948, que en su artículo 21 señala: "La voluntad del pueblo es la base de la autoridad del poder público; esta voluntad se expresará mediante elecciones auténticas que habrán de celebrarse periódicamente, por sufragio universal e igual y por voto secreto $u$ otro procedimiento equivalente que garantice la libertad de voto".

De ahí, que el sistema elegido deba facilitar la expresión de la voluntad del pueblo, mediante la celebración de elecciones periódicas y auténticas; es decir, libres y competitivas, realizadas con base en el sufragio universal, en la igualdad del poder del voto y en el escrutinio secreto. (Arnaldo, 1999: 17).

Pero, llegados a este punto, parece conveniente diferenciar entre elecciones no competitivas y elecciones competitivas:

Con el nombre de elecciones no competitivas definimos aquellos sistemas electorales, establecidos en función de los intereses de algunas de las partes contendientes, que reducen o eliminan de hecho, la posibilidad de una alternativa efectiva en la selección de titulares de poder.

El hecho de hacer mención a estas elecciones no competitivas o "elections without choice", en terminología anglosajona, se debe a dos razones:

La primera, porque los países actualmente reconocidos como democráticos incluyen en sus pasados períodos- a veces muy prolongadoselecciones no competitivas, en las que el acceso al sufragio o la igualdad estuvieron legal y prácticamente disminuidos.

La segunda, porque en el mundo de hoy aún nos encontramos con la existencia de elecciones no competitivas o semicompetitivas y en número superior a las que pueden considerarse como aceptablemente competitivas.

Sin embargo, vamos a analizar los sistemas electorales $^{3}$ que dan lugar a elecciones competitivas y para ello, enumeraremos los principios o requisitos, tanto legales como socioeconómicos, que las definen. Cuanto mayor sea el grado de cumplimiento de dichos requisitos, mayor grado de garantía se otorgará a los participantes en el proceso electoral, en relación con la seguridad, la libertad y la igualdad. Ellos son:

- Derecho universal al sufragio.

- Convocatoria regulary periódica de elecciones.

3 Por ser el elemento clave, tal y como hemos enunciado anteriormente, del Estadodemocrático liberal. 
- Libertad de asociación para presentar candidatos.

- Igualdad tendencial de oportunidades de los candidatos para el acceso a los medios de información y publicidad.

- Neutralidad del gobierno en la organización del proceso electoral.

- Garantías para una libre emisión de voto (ausencia de coacciones, protección del secreto).
- Recuento público de los votos emitidos.

- Aplicación de las reglas preestablecidas para adjudicar los escaños entre los candidatos.

- Existencia de una instancia independiente para dirimir los conflictos suscitados por la aplicación de las normas electorales.

\section{LA FÓRMULA ELECTORAL}

Se entiende por fórmula electoral el procedimiento de cálculo que convierte las preferencias expresadas por los electores, en una distribución de escaños entre los diversos candidatos o candidaturas que se presentan en un distrito determinado. (Vallés y Bosch, 1997:83).

Las preferencias de los ciudadanos se plasman en los resultados electorales, expresados en número de votos, y correspondea lafórmula electoral traducir esas preferencias individuales en decisiones colectivas, asignándoles un determinado número de los denominados escaños.

Las fórmulas electorales ${ }^{4}$ se clasifican en: mayoritarias y proporcionales.

Las fórmulas mayoritarias, que son las más antiguas, actúan asignando como vencedor a aquel candidato que consigue el mayor número de votos. De esta manera, resuelven el problema de traducir votos conseguidos por el candidato o lista de candidatos en número de escaños.

Se trata de una fórmula cuyo principal objetivo es distinguir entre vencedores y perdedores y, por ello,: basta la diferencia de un solo voto para ganar o perder. De ahí, que quien no forma parte de la mayoría, queda excluido del juego electoral.

Las fórmulas proporcionales pretenden distribuir los escaños entre las candidaturas presentadas en función del número de votos obtenidos por cada una de ellas: se trata de determinar, en el denominado juego electoral, el pago en número de votos que cuesta un escaño.

La denominación de fórmula proporcional está más extendida que la de fórmula distributiva,

4 La elección de una u otra fórmula electoral, ha permitido clasificar los diferentes sistemas electorales en mayoritarios y proporcionales, si bien, otros estudios han restado importancia a este elemento como definitorio de los sistemas electorales, una vez que se han analizado en diversos contextos y en relación con otros elementos del propio sistema. (Vallés y Bosch, 1997:84). 
aunque resulta más precisa esta última, por cuanto, el carácter proporcional de la fórmula sólo se alcanza dependiendo de la presencia e importancia de otros elementos, tales como la magnitud de la circunscripción, umbral mínimo de votos, etc.

En las fórmulas proporcionales, el conjunto de los electores participa en la decisión sobre el reparto de puesto y ésta es compartida de modo tendencial por ellos mismos. Por tanto, la expresión política de la totalidad de los electores está integrada en la representación elegida.

La diferencia más importante entre ambos tipos de fórmulas radica en que en las mayoritarias el acento se pone en el principio representaciónmandato, entendido como delegación claramente otorgada a un grupo o partido, que es aquel que ha conseguido mayor apoyo, según el número de votos obtenidos. Por su parte, las fórmulas proporcionales o distributivas, atienden más al objetivo de conseguir la representatividad del conjunto de los elegidos.

\subsection{Evolución histórica del empleo de fórmulas electorales}

En los primeros regímenes representativos de corte liberal se aplicaban fórmulas de tipo mayoritario, en las que los electores, que suponían una pequeña parte de la población adulta, daban su confianza a los elegidos para que les gobernaran. Los países pioneros en este tipo de fórmulas fueron Gran Bretaña y Estados Unidos.

Un elemento importante y determinante por considerar en esta evolución del empleo de fórmulas electorales, fue la paulatina ampliación del sufragio, que puso fin a una situación de monopolio electoral, en la que sólo participaban los grupos sociales dominantes. De esta manera, otras capas de población salen al escenario y emerge la exigencia del "principio de representatividad" que sólo las fórmulas proporcionales pueden ofrecer.

Las primeras propuestas de fórmulas distributivas se sitúan a mediados del siglo XIX, y toman el nombre de sus precursores: el danés Andrae (1855) y el británico Hare (1857). El debate político y teórico sobre las correspondientes ventajas y desventajas de cada uno de los principios políticos y de cada una de las fórmulas que los encarnan es y ha sido uno de los temas permanentes en la literatura política y constitucional.

Los principales defensores de una y otra línea en el siglo XIX fueron Walter Bagehot - a favor del mandato mayoritario-, quien otorgó como ventaja principal la determinación de una mayoría parlamentaria a la que se confía un mandato claro para desarrollar una determinada política gubernamental y John Stuart Mill - defensor de las fórmulas proporcionales- con el fin de conseguir una Cámara que fuera reflejo aproximado de las tendencias existentes en el cuerpo electoral, incluyendo representaciones de cada una de ellas en proporción a su fuerza respectiva.

\subsection{Las fórmulas mayoritarias. Variantes}

\subsubsection{Mayoría simple}

En la fórmula mayoritaria de mayoría simple se proclama vencedor al candidato que obtiene más votos. No importa el número de votos que separe al vencedor de los vencidos. Sea esta diferencia de un voto o de varios miles, los vencidos quedan, de igual modo, excluidos del equipo de gobierno. 
La tradición anglosajona la denomina como "first-past-the-post", que en una traducción aproximada significa "el primero que cruza la meta, gana". Es la fórmula más usual en el mundo anglosajón.

\subsubsection{Mayoría absoluta}

En esta variante de fórmula mayoritaria se exige la mitad más uno de los votos para alzarse como vencedor. Con ello, se refuerza el apoyo electoral exigido a un grupo y resulta más aceptable su elección, ante los perdedores.

Con el fin de favorecer la consecución de una mayoría absoluta, difícil de obtener, se formulan dos modalidades de voto:

\subsubsection{La doble votación (o las dos vueltas)}

En el caso de que una vez celebrada la elección, ningún candidato hubiera obtenido mayoría absoluta, se repite la votación en la denominada segunda vuelta. Por norma general, la participación en la segunda vuelta queda restringida tan sólo a aquellos candidatos que han obtenido cierto número de votos en la primera, tal y como sucede en el actual sistema francés; o a los dos candidatos más votados en esa primera votación.

Esta forma de proceder, fomenta la retirada voluntaria de alguno o algunos de los candidatos, con el objetivo de que sus votos refuercen en la segunda vuelta, las posibilidades de otros candidatos afines y mejor clasificados. Esta retirada tiene un coste y suele ser objeto de negociaciones y compromisos entre candidatos y partidos, que establecen estrategias de apoyos y retiradas en la fase previa a las elecciones o entre las dos vueltas y tras analizar los resultados obtenidos en la primera votación.

\subsubsection{El voto alternativo o preferencial}

En esta variante, el votante indica en primer lugar el nombre de su candidato preferido y, a continuación, señala un orden de prioridad entre los demás candidatos presentados. En el caso de que uno obtenga la mayoría absoluta de las primeras preferencias expresadas, es proclamado vencedor.

En caso de no ser así, se elimina al candidato con menor número de votos, a la vez que se traspasan, a sus respectivos destinatarios, las segundas preferencias manifestadas en los votos recibidos por el candidato eliminado. Se repite el procedimiento hasta que uno de ellos consigue reunir un número de preferencias equivalentes a la mayoría absoluta requerida.

Con este procedimiento se pretende reunir en una sola vuelta o votación las dos operaciones del procedimiento anterior, la denominada segunda vuelta.

\subsection{La fórmulas distributivas variantes}

Las fórmulas distributivas tratan de asignar el número de escaños en función de la cantidad de votos obtenidos por cada partido o candidato. Para ello, es necesario establecer el número de votos que da derecho a un escaño o en otras palabras, "el precio, en términos de votos, quecuesta un escaño" y a partir de ahí, calcular el número de puestos que corresponderá a cada partido.

Con el fin de proceder a su mejor explicación, denominamos:

- m - por magnitud-, al número de escaños de la circunscripción.

- $v$ al total de votos válidos emitidos por la misma y 
- q a la cuota o número de votos necesarios para conseguir un escaño.

$$
\text { De ahí que } \mathrm{q}=\mathrm{v} / \mathrm{m} .^{5}
$$

La adjudicación de los escaños se establece mediante la división de los votos obtenidos por cada candidatura vA por la cuota o cociente electoral, q, para conseguir el número de escaños que le corresponde eA. Esta división, muy rara vez, tendrá como resultado un número entero; ofreciendo casi siempre será un número racional.

En este punto se procede a adjudicar los escaños en función de los números enteros que obtiene cada partido y queda pendiente la asignación de los escaños restantes y la aplicación de las fracciones de cada partido. Para resolver este problema, se presentan dos alternativas: la fórmula del resto mayor y la fórmula de la media mayor.

\subsubsection{La fórmula del resto mayor}

- El cálculo de la cuota y su aplicación. En esta variante, el criterio para la asignación de escaños no atribuidos en una primera distribución se basa en la magnitud del resto de sufragios no utilizados de cada una de las candidaturas.

Con el fin de proceder a la atribución de escaños, se determina la cuota o cociente electoral, que da derecho a un escaño. Esta cuota es el resultado de dividir el total de votos emitidos por:

- El número de escaños a proveer en el correspondiente distrito $o$

- Por este mismo número aumentado en una o

- Por este mismo número aumentado más unidades ${ }^{6}$.

Cuadro 1. Cálculo de la cuota electoral en variantes de la fórmula distributiva del resto mayor

\begin{tabular}{|c|c|}
\hline VARIANTES & CÁLCULODELACUOTA \\
\hline Hare & $\mathrm{q}=\mathrm{v} / \mathrm{m}$ \\
\hline Droop (Hagenbach-Bischof) & $\mathrm{q}=\mathrm{v} / \mathrm{m}+1$ \\
\hline Imperiali & $\mathrm{q}=\mathrm{v} / \mathrm{m}+2$ \\
\hline Imperial reforzada & $\mathrm{q}=\mathrm{v} / \mathrm{m}+3$ \\
\hline
\end{tabular}

Fuente: Vallés y Bosch. 1997,90

5 Dicha cuota es denominado cociente electoral, puesto que es la cantidad que resulta de la división entre votos y escaños de un distrito.

6 Cada una de las variantes toma el nombre de sus proponentes. Así, sus denominaciones son: Cuota de HareNiemeyer, Cuota de Drop y Cuota de Imperiali. 
Una vez que se ha determinado la correspondiente cuota, se procede a atribuir a cada lista, tantos escaños como veces se contiene la cuota o cociente electoral, en el total de votos recibidos por dicha lista. Efectuada esta operación y, en caso de que todavía queden puestos por cubrir, será la magnitud de los votos sobrantes o resto de cada lista- expresada en la fracción de la cuota- lo que decida la distribución de estos escaños sobrantes. Para ello, estos escaños pendientes se irán asignando a las listas que presenten la fracción más elevada de la cuota - equivalente al resto mayor-.

\section{- La variante Hare-Niemeyer}

Esta variante responde a la misma lógica de la fórmula del resto mayor con cuota Hare. El proceso de cálculo es diferente, aunque produce el mismo resultado. Para obtener la cuota, se multiplica primero el número de votos conseguido por cada partido por la magnitud .o número de escaños del distrito-. Posteriormente, se divide la cantidad resultante de este producto por el total de votos válidos emitidos por el distrito. La cuota por partido, así obtenida, informa del número de escaños asignados a cada candidatura, distribuyendo, en primer lugar, los correspondientes a cuotas enteras y asignando los restantes a los partidos con mayores fracciones de cuotas, equivalentes a mayores restos.

\section{- La variante Hare-Andrae}

Constituye la expresión más antigua de la fórmula proporcional, puesto que sus autores la esbozaron, casi a la vez, a mediados del siglo XIX: el hacendista danés Carl G. Andrae en 1855 y el abogado londinense Thomas Hare en 1857.
En primer lugar, se establece la cuota necesaria para obtener un escaño. Cada elector vota por su candidato preferido y a continuación procede a marcar el orden de preferencia entre los demás aspirantes.

Los inmediatamente elegidos son aquellos, que una vez efectuado el recuento de las primeras preferencias obtenidas por cada uno de los candidatos, alcanzan dicha cuota. Si después de esta primera adjudicación, siguen vacantes uno o algunos escaños, los sufragios excedentes-que rebasan la cuota- a favor de los candidatos ya elegidos, se adjudican a los candidatos que figuran como segunda preferencia del elector.

En el caso de que el candidato elegido no disponga de sufragios excedentes, serán las segundas preferencias del candidato situado en último lugar las que se utilizarán para favorecer a otros en mejor situación. En ambos casos, se transfieren los votos no utilizados para su primer beneficiario -que ya ha obtenido su escaño o que no tiene ninguna posibilidad de hacerlo-. Los votos transferidos serán útiles para que otros candidatos alcancen la cuota y puedan ser elegidos. Cuando después de esta segunda etapa de asignación permanecen todavía algunos escaños por proveer, se repite la operación tantas veces como sea necesario, hasta completar el número de escaños.

Para comprender mejor la relativa complejidad de este método, es preciso compararlo con un sistema de votaciones sucesivas que se van repitiendo hasta que un número de candidatos, igual al de puestos en disputa, consigue la cuota de votos requerida. Veamos un ejemplo: 
Lakeman (1974:114) refiere como ejemplo la elección de un comité de clase por los alumnos de una escuela. "Los alumnos debían elegir un comité, situándose junto al muchacho que preferían. En un primer movimiento, se creaban una serie de grupos desiguales. Pero, a continuación, los alumnos de los grupos más numerosos llegaban a la conclusión que no todos ellos eran necesarios para la elección de su favorito y algunos se trasladaban de un grupo a otro para ayudar a otro candidato. Por otra parte, los escasos partidarios de un muchacho poco popular daban por perdida su candidatura y se situaban junto a otro que les parecía con más posibilidades. El resultado final era que un número de candidatos igual al número de miembros del comité quedaban rodeados por la misma cantidad de partidarios. Sólo dos o tres muchachos, descontentos con los elegidos, quedaban al margen de los grupos".

Una de las ventajas que se desprende de esta variante estriba en la disminución de votos desperdiciados o no empleados, puesto que tienen igual consideración las preferencias de los electores cuyo primer elegido resulta ganador y las preferencias de los electores que hayan votado a un candidato con exceso de votos.

Al mismo tiempo, los electores no están obligatoriamente vinculados a listas de partido y pueden, en teoría, combinar sus preferencias, dedicándolas a candidatos de partidos diferentes. (Vallés y Bosch: 1997, 93).

\subsubsection{La fórmula de la media mayor}

Con la aplicación de esta fórmula se pretende principalmente conseguir que el coste medio (medido en votos) que se debe pagar por conseguir un escaño, sea idéntico para cada partido. Con este fin, cada uno de los escaños va siendo sucesivamente atribuido al partido que presenta una media más elevada de votos por escaño.(Vallés y Bosch, 1997:94).

La versión más simple de esta fórmula es la siguiente:

- En una primera fase, se establece la cuota o cociente electoral, dividiendo el total de votos emitidos en el distrito por el número de diputados por elegir: $q=v / m$.

- A continuación, se procede a una primera atribución de escaños, otorgando a cada partido, tantos escaños, cuantas veces se contiene la cuota en su total de sufragios.

Si tras esta primera fase, permanecen escaños sin provisión y los partidos conservan votos sobrantes, se procede a una segunda distribución. Para ello, los votos de cada partido se dividen por el número de puestos que ya ha conseguido en la primera fase más uno, con el fin de averiguar cuál sería ahora su media de votos por escaño, si se le atribuyera el puesto pendiente de provisión.

La lista que presenta una media mayor, se hace con el escaño. Si permanece todavía algún escaño no provisto, se procede de nuevo a obtener las medias de cada candidatura, de acuerdo con la operación descrita. $Y$ así sucesivamente, hasta distribuirlos todos.

En relación con la fórmula del resto mayor, esta favorece a los partidos con mayor número de votos y perjudica a los minoritarios, puesto que son los primeros los que suelen presentar mayores medias en las sucesivas rondas. 


\section{- La variante d’Hont}

Recibe su nombre del profesor belga que la propuso. Se entiende como una superación en relación con la operativa descrita, puesto que trata de reunir en una sola operación todas las etapas descritas en el proceso anterior.

Para ello, se procede dividiendo sucesivamente el número de sufragios de cada candidatura por 1-2-3-4..., hasta el número total de escaños por cubrir. A continuación, los cocientes resultantes de las divisiones anteriores se ordenan de mayor a menor, en una serie que ha de comprender tantos cocientes como escaños en disputa.

A partir de ese momento, las disposiciones electorales adoptan uno de los dos siguientes caminos:

- El primero, previsto en la ley española y portuguesa, atribuye los escaños a los partidos que presentan mayores cocientes de la lista.

- El segundo, en el caso de los belgas y, en el supuesto de un distrito con cinco escaños, se localiza el quinto de los cocientes de la serie ordenada de mayor a menor. Este cociente es denominado "divisor común". El total de votos obtenidos en el Distrito por cada partido es dividido por el divisor común: el resultado de esta división nos da el número de diputados que corresponde a cada lista de partido.

\section{- La variante Sainte-Laguë}

Trata de restar la ventaja relativa que obtienen los grandes partidos, cuando se aplica la fórmula de la media mayor.
La corrección afecta a los divisores -1,2,3...que producen la serie de medias. La modificación más conocida es la que recibe el nombre de variante Sainte-Laguë. En esta ocasión, la secuencia de divisores se sustituye por la serie de números impares: $1,3,5,7, \ldots$. De este modo, al incrementar la diferencia entre divisores, también se incrementa la diferencia entre cocientes. Con ello, los partidos mayores ven relativamente disminuida su ventaja, pues resulta más elevado para ellos el pago que realizan por cada nuevo escaño.

Sin embargo, y, con el fin de no beneficiar demasiado a los partidos más pequeños, el primer divisor de la serie se sustituye por 1,4. De este modo, aumenta un $40 \%$ el precio del primer posible escaño de un partido con pocos sufragios. Esta variante se conoce como Sainte-Laguë corregida y se utilizó en algunos países escandinavos en los años 50.

\section{- La variante Imperiali}

Los divisores empleados en esta variante son $2,3,4,5, \ldots$ y al excluir el primer divisor igual a 1 , se hace muy difícil obtener el primer escaño a los partidos con menos votos y aumenta el peso de los partidos mayores. Con ello, se pretende beneficiar a los partidos mayoritarios.

\section{- La variante Hagenbach-Bischoff}

Esta variante se basa en la media mayor y se utiliza la cuota Droop. Se aplica actualmente en Suiza, Luxemburgo y Lichtenstein.

La asignación de escaños se produce en dos fases:

- En la primera, se obtiene la ya mencionada cuota electoral, dividiendo el total de votos válidos emitidos por el número de escaños más uno: $\mathrm{q}=\mathrm{v} / \mathrm{m}+1$. 
- Posteriormente, se divide el total de votos de cada partido por esta cuota y se obtiene así el número de escaños que corresponde a cada partido.

Si tras esta operación, permanecen escaños sin asignación, el primero de los escaños pendientes se adjudica a la candidatura que presente el cociente más alto, una vez dividido el número de votos obtenido por el número de escaños que ya le hubieren sido asignados en la primera distribución incrementado en una unidad. Se procede nuevamente de este modo hasta completar la asignación de todos los puestos.

Los resultados obtenidos son muy parecidos a los de la Ley de d'Hont.

\section{APLICACIÓN COMPARADA DE FÓRMULAS DISTRIBUTIVAS}

continuación procedo a aplicar las distintas fórmulas electorales a un resultado electoral real.

Magnitud del distrito: 13 concejales.

Votos emitidos: 3598.

Partidos recurrentes: $1,2,3,4,5,6,7$ y 8 .

En todos los casos, se procede de la misma manera: en primer lugar, se define la cuota y a continuación se distribuyen los escaños, en función de la variante empleada.

\section{FóRMULA DE LA MEDIA MAYOR}

- Cálculo de la cuota : v/m = $3598 / 13=$ 276,7692 .

- Distribución de escaños, según división de votos de cada partido por la cuota según el resto mayor:

\begin{tabular}{|c|c|c|c|c|c|}
\hline Partido & No. votos & $\begin{array}{l}\text { No.votos } \\
1 \text { cuota }\end{array}$ & $\begin{array}{l}\text { Escaños según } \\
\text { cuota entera }\end{array}$ & $\begin{array}{l}\text { Escaños según } \\
\text { resto mayor }\end{array}$ & $\begin{array}{l}\text { Escaños por } \\
\text { partido }\end{array}$ \\
\hline 1 & 861 & 3,1108 & 3 & & 3 \\
\hline 2 & 732 & 2,6448 & 2 & & 2 \\
\hline 3 & 570 & 2,0594 & 2 & & 2 \\
\hline 4 & 528 & 1,9077 & 1 & 1 & 2 \\
\hline 5 & 435 & 1,5717 & 1 & & 1 \\
\hline 6 & 209 & 0,7551 & 0 & 1 & 1 \\
\hline 7 & 183 & 0,6612 & 0 & 1 & 1 \\
\hline 8 & 80 & 0,2890 & 0 & 1 & 1 \\
\hline Total & 3598 & & 9 & 4 & 13 \\
\hline
\end{tabular}




\section{FÓRMULA DE LA MEDIA MAYOR}

Variante de d'Hont (divisores: 1-2-3-4-5...).

- Atribución de los escaños a los partidos que presentan los cocientes mayores hasta proveer los 13 escaños. Los cocientes

- División del número de votos de cada partido mayores aparecen en negrita. por los divisores.

\begin{tabular}{|c|c|c|c|c|c|c|}
\hline Partido & No. votos & No.votos/1 & No.votos/2 & No.votos/3 & No.votos/4 & $\begin{array}{l}\text { Escaños } \\
\text { por partido }\end{array}$ \\
\hline 1 & 861 & 861 & 430,5 & 287 & 215,25 & 4 \\
\hline 2 & 732 & 732 & 366 & 244 & 183 & 3 \\
\hline 3 & 570 & 570 & 285 & 190 & 142,5 & 2 \\
\hline 4 & 528 & 528 & 264 & 176 & 132 & 2 \\
\hline 5 & 435 & 435 & 217,5 & 145 & 108,75 & 2 \\
\hline 6 & 209 & 209 & 104,5 & 69,67 & 52,25 & \\
\hline 7 & 183 & 183 & 91,5 & 61 & 45,75 & \\
\hline 8 & 80 & 80 & & & & \\
\hline Total & 3598 & & & & & 13 \\
\hline
\end{tabular}

Variante Sainte-Laguë (divisores: 1-3-5-7 ...).

- División del número de votos de cada partido por los divisores.
- Atribución de los escaños a los partidos que presentan los cocientes mayores hasta proveer los 13 escaños. Los cocientes mayores aparecen en negrita.

\begin{tabular}{|c|c|c|c|c|c|c|}
\hline Partido & No. votos & No.votos/1 & No.votos/3 & No.votos/5 & No.votos/7 & $\begin{array}{c}\text { Escaños } \\
\text { por partido }\end{array}$ \\
\hline 1 & 861 & $\mathbf{8 6 1}$ & $\mathbf{2 8 7}$ & $\mathbf{1 7 2 , 2}$ & 123 & 3 \\
\hline 2 & 732 & $\mathbf{7 3 2}$ & $\mathbf{2 4 4}$ & $\mathbf{1 4 6 , 4}$ & 104,57 & 3 \\
\hline 3 & 570 & $\mathbf{5 7 0}$ & $\mathbf{1 9 0}$ & 114 & 81,42 & 2 \\
\hline 4 & 528 & $\mathbf{5 2 8}$ & $\mathbf{1 7 6}$ & 105,6 & 75,42 & 2 \\
\hline 5 & 435 & $\mathbf{4 3 5}$ & 145 & 87 & 62,14 & 1 \\
\hline 6 & 209 & $\mathbf{2 0 9}$ & 69,7 & 41,8 & 29,85 & 1 \\
\hline 7 & 183 & $\mathbf{1 8 3}$ & 61 & 36,6 & 26,14 & 1 \\
\hline 8 & 80 & 80 & 26,7 & 16 & 11,42 & \\
\hline Total & 3598 & & & & & 13 \\
\hline
\end{tabular}


Variante Sainte-Laguë corregida (divisores: 1,4-

3-5-7...).

- División del número de votos de cada partido por los divisores.
- Atribución de los escaños a los partidos que presentan los cocientes mayores hasta proveer los 13 escaños. Los cocientes mayores aparecen en negrita.

\begin{tabular}{|c|c|c|c|c|c|c|}
\hline Partido & No. votos & No.votos/1,4 & No.votos/3 & No.votos/5 & No.votos/7 & $\begin{array}{c}\text { Escaños } \\
\text { por partido }\end{array}$ \\
\hline 1 & 861 & 615 & 287 & 172,2 & 123 & 3 \\
\hline 2 & 732 & 522,85 & 244 & 146,4 & 104,57 & 3 \\
\hline 3 & 570 & 407,14 & 190 & 114 & 81,42 & 2 \\
\hline 4 & 528 & 377,14 & 176 & 105,6 & 75,42 & 2 \\
\hline 5 & 435 & 310,71 & 145 & 87 & 62,14 & 2 \\
\hline 6 & 209 & 149,28 & 69,7 & 41,8 & 29,85 & 1 \\
\hline 7 & 183 & 130,71 & 61 & 36,6 & 26,14 & \\
\hline 8 & 80 & 57,14 & 26,7 & 16 & 11,42 & \\
\hline Total & 3598 & & & & & 13 \\
\hline
\end{tabular}

Variante Imperiali (divisores: 2-3-4-5-6...)

- División del número de votos de cada partido por los divisores.
- Atribución de los escaños a los partidos que presentan los cocientes mayores hasta proveer los 13 escaños. Los cocientes mayores aparecen en negrita.

\begin{tabular}{|c|c|c|c|c|c|c|}
\hline Partido & No. votos 1 & No.votos/2 & No.votos/3 & No.votos/4 & No.votos/5 & $\begin{array}{l}\text { Escaños } \\
\text { por partido }\end{array}$ \\
\hline 1 & 861 & 430,5 & 287 & 215,25 & 172,2 & 4 \\
\hline 2 & 732 & 366 & 244 & 183 & 146,4 & 3 \\
\hline 3 & 570 & 285 & 190 & 142,5 & 114 & 2 \\
\hline 4 & 528 & 264 & 176 & 132 & 105,6 & 2 \\
\hline 5 & 435 & 217,5 & 145 & 108,75 & 87 & 2 \\
\hline 6 & 209 & 104,5 & 69,7 & 52,25 & 41,8 & \\
\hline 7 & 183 & 91,5 & 61 & 45,75 & 36,6 & \\
\hline 8 & 80 & & 26,7 & & 16 & \\
\hline Total & 3598 & & & & & 13 \\
\hline
\end{tabular}


Tabla comparativa de la distribución de escaños según las fórmulas aplicadas

\begin{tabular}{|c|c|c|c|c|c|}
\multirow{2}{*}{$\begin{array}{c}\text { Periodos } \\
\text { concurrentes }\end{array}$} & $\begin{array}{c}\text { No. escaños } \\
\text { según.Resto } \\
\text { mayor }\end{array}$ & \multicolumn{4}{|c|}{ No. de escaños según: media mayor } \\
\cline { 3 - 6 } & D'Hont & Sainte-Lagué & $\begin{array}{c}\text { Sainte-Lagué } \\
\text { corregida }\end{array}$ & Imperiali \\
\hline 1 & 3 & 4 & 3 & 3 & 4 \\
\hline 2 & 2 & 3 & 3 & 3 & 3 \\
\hline 3 & 2 & 2 & 2 & 2 & 2 \\
\hline 4 & 2 & 2 & 2 & 2 & 2 \\
\hline 5 & 1 & 2 & 1 & 2 & 2 \\
\hline 6 & 1 & 0 & 1 & 1 & 0 \\
\hline 7 & 1 & 0 & 1 & 0 & 0 \\
\hline 8 & 1 & 0 & 0 & 0 & 13 \\
\hline & 13 & 13 & 13 & 13 & \\
\hline
\end{tabular}




\section{ConClusiones}

T

ras analizar dos de los tres elementos fundamentales del régimen o sistema electoral, en concreto, como son, la forma de expresión del voto y la fórmula repartidora y variantes, las conclusiones son básicamente dos:

La primera, coincide con la opinión de algunos estudiosos, respecto a que el sistema electoral, por su propia naturaleza, debe ser considerado no como un instrumento científico de acción neutra, sino como una herramienta que, en función de la variante elegida va a arrojar resultados diferentes y, por tanto, resulta susceptible de ser manejada por conveniencias políticas, fundamentalmente porque va a dar lugar a diferentes pactos a la hora de formar gobierno.

Esta conclusión es fácilmente observable, analizando los resultados que aparecen en la tabla comparativa anterior, donde se muestra la distribución de escaños aplicados a unos resultados electorales concretos.

De acuerdo con el grado de representatividad conseguido, la clasificación de las fórmulas aplicadas, de mayor a menor, es:

- Según la fórmula distributiva del resto mayor: todos los partidos políticos concurrentes, ocho de ocho, obtienen representación. Esta situación de máxima representatividad sólo se obtiene aplicando dicha fórmula.

- De acuerdo con la fórmula distributiva de la media mayor en su variante Sainte-Laguë, siete de ocho partidos concurrentes, obtienen representación.
- La fórmula distributiva de la media mayor en su variante Sainte-Lagué corregida, ofrece representación a seis de los ocho partidos concurrentes.

- Las fórmulas d'Hont e Imperiali ofrecen idénticos resultados en cuanto a la adjudicación de escaños; obtienen representación cinco de los ocho partidos concurrentes.

En segundo lugar y, a la hora de formar un gobierno estable, que presupone la presencia de mayoría absoluta, (en el caso de nuestro ejemplo, conseguir siete escaños de un total de trece) $y$, teniendo en cuenta, los resultados obtenidos, los pactos posibles también difieren, pero en la misma línea que apuntamos en la primera conclusión: las posibilidades de pacto aumentan cuando se consigue un mayor grado de representatividad.

De ahí, que la clasificación, de mayor a menor, en cuanto a pactos de gobernabilidad, coincide con la planteada en la primera conclusión:

- Con la aplicación de la fórmula del resto mayor, el número de posibilidades de pactos es mayor que en ninguna otra.

- La fórmula distributiva de la media mayor en su variante Sainte-Laguë.

- La fórmula distributiva de la media mayor en su variante Sainte-Lagué corregida.

- Las fórmulas d'Hont e Imperiali aportan idénticos resultados. 


\section{Bibliografía}

- ARNAldo, E., El régimen electoral en España. Centro de Estudios Políticos y Constitucionales. BOE. Madrid. 1999

- BILBAO, J.M."Sistema Electoral y alternancia en España".Documento. Universidad de Sevilla.

- GARCIA-PELAYO, M. "Derecho Constitucional Comparado".Ciencias Sociales. Alianza Editorial. Madrid. 2000.

- GONZALEZ, J.C. Derecho electoral español. Normas y Procedimientos. Tecnos. Madrid. 1996.
- LLERA, F ."Gobernabilidad y sistema de partidos: dimensión territorial e identitaria".Cuadernos del Pensamiento Político $N^{\circ}$ 3. FAES Junio-Septiembre 2004.(19-48).

- REINOSO, D. Europa y los sistemas electorales. Fundación Universidad-Empresa. Madrid. 1990.

- SANTOLAYA, P. Manual de Procedimiento Electoral. $3^{a}$ Edición. Ministerio de Justicia e Interior. Secretaría General Técnica. 1995.

- VAlLes, ,J. y BOSCH, A. Sistemas electorales y gobierno representativo. Ariel Ciencia Política. Barcelona. 1997. 


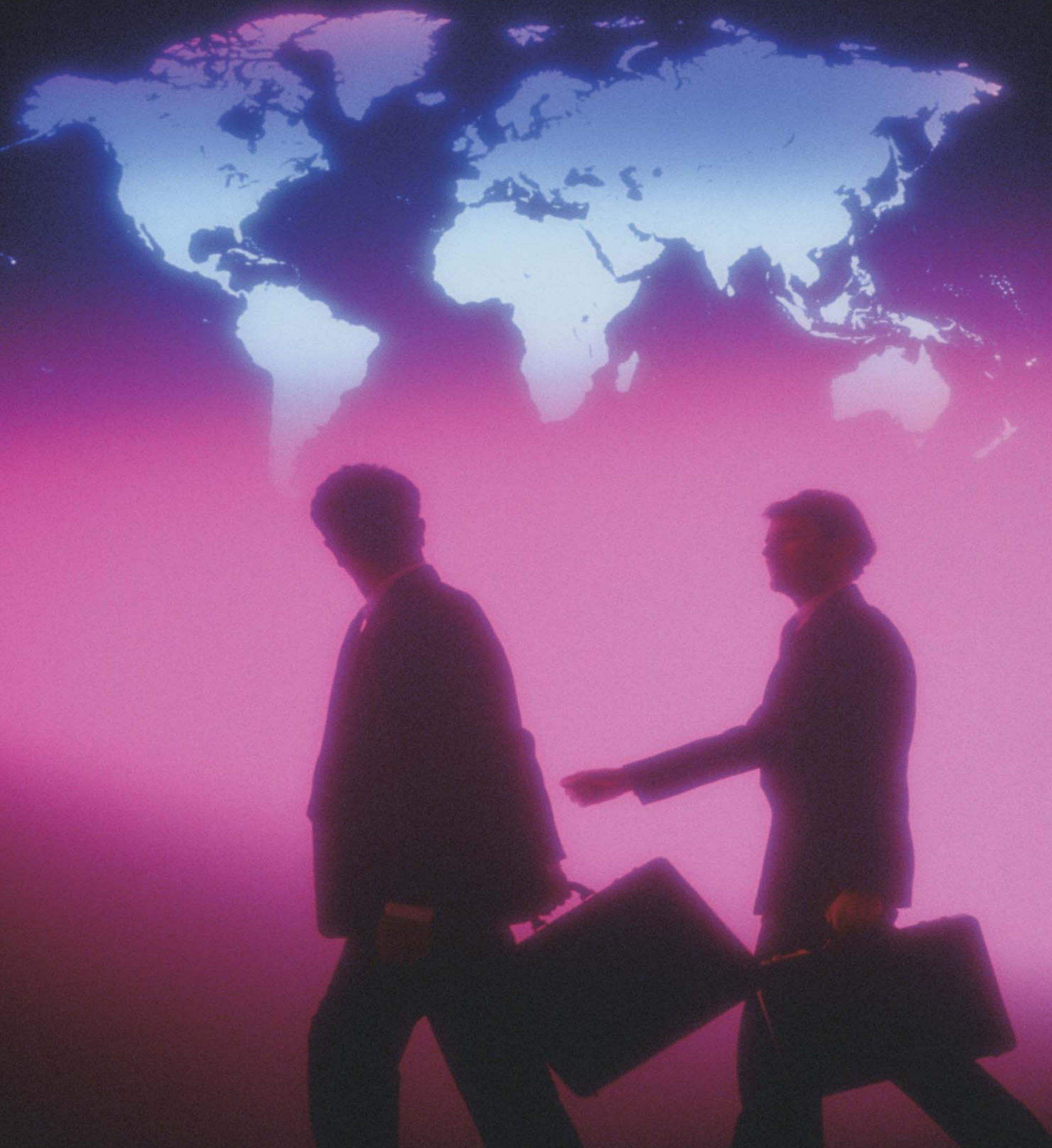

